\title{
Inventory Management to Secure Software System
}

\author{
Jung Hyun Kim1), Sarita Singh Bhadauria2)
}

\begin{abstract}
The agile methodologies approach was being popular as a result of of its usage within the evolution of stock in numerous things. The potential threats to computer based mostly system were solved and determine by exploitation techniques of agile. The result it shows is how agile methodologies work effectively on inventory systems. At present there area unit numerous network securities or package methodologies measure like management tools area unit employed in systems. The main problem is to seek out in what manner inventory management is being organized so as to operate expeditiously and maintaining security the answer to the matter has been mentioned for the decades and additionally we've seen several remedies to the current issue. The above drawback will be solved by exploitation agile methodologies. The complete work relies on a technique that analyzes package exploitation a number of software techniques like mythical being, eclipse in field of web technologies. The values area unit the inputs to the system however here the values are taken from markup language pages to these pages we want to high offer confidentiality and security that gains package security goals.
\end{abstract}

Keywords : agile, exploitation, security, confidentiality, Software, Security.

\section{Introduction}

Now-a-day, many national wide super marketing companies are facing trouble in managing software and in managing goods[1]. Inventory management software depends on stocks that it import and export and the transactions it do online. The software need to take record of daily, monthly and yearly production[2-4]. To maintain all these records it need a high level security mechanism i.e., providing security to the data that involves individual, unit and customers of system[5-9]. In addition to this, the many network attack we need to face and is also increasing day by day quickly. Because of this the security risk for the information system facing is increasing day to day. At present there are various network securities or software methodologies measure like management tools are used in systems. The main problem is to

Received(July 8, 2016), Review Result(1st: July 29, 2016, 2015, 2nd: August 29, 2016), Accepted(September 10, 2016)

${ }^{1}$ Conversing Technology, Hoseo Graduate School of Venture, Seoul email: hyun2@hanmail.net

${ }^{2}$ (Corresponding Author) Department of Computer Science and Engineering, KL University email: saritamits61@yahoo.co.in 
find in what way inventory management is being organized in order to function efficiently and maintaining security the solution to the problem has been discussed for the decades and also we have seen many remedies to this issue. The above problem can be solved by using agile methodologies.

As we have discussed that managing problem is the main issue that are involved in computer based system which are growing more in number this causes a serious problem in stock. This problem has started when human has started managing the work manually in those days human write records manually as there is increase in population there started to invent a software which manages all the records there were also successful in inventing a software using traditional approach which has solved many problem that human is facing. The methodology at present companies are using is agile methodologies.

The agile methodologies approach was being popular because of its usage in the evolution of stock in different situations. The potential threats to computer based system were solved and identify by using techniques of agile. The result it shows is how agile methodologies work effectively on inventory systems.

At present security of software systems is plays major role in many organization and all are depending on them to provide efficient security for their data and also to manage the data properly. The need to analyze and determine the risks we face is of emerging software systems because we already know that there is no software that provides complete security and management till now. The complete work is based on a methodology that analyzes software using some of software techniques like Ajax, eclipse in field of web technologies. The values are the inputs to the system but here the values are taken from html pages to these pages we need to high provide confidentiality and security that gains software security goals.

Agile methodology is widely accepted, in this we need to provide security to the information. From the collected text we can analyze all the risks that we face. All means we can identify risks we face either from system software or from network software etc. it is being faced from past. This techniques is used for any kind of inventory system and also used in many business applications

Here we explain you how agile is developed and in what way inventory is created. To build software we can use any web based language which are helpful to create static and dynamic web pages and run in web browser. To these software is connected with databases and dumped into browser. Inventory a stock management handles the data so while building a project we need specify the capacity of it also so that disputes don't occur. 


\section{Related Works}

\subsection{Rationale}

We can manage data by using artificial intelligence also but we adopt software engineering only because it is easy and helpful. It plays a crucial role in development of any software so it is very hard to develop any software without the help of software engineering. Our topic is based on inventory management. A software process is a collection of activities, task and transformations, finalization which results in collection of software and similar products. Though many of organizations subject new ideas for the decrease of problems in inventory they are using only agile process in inventory because agile can handle dynamic changes and make continuous checking on data, as software process model mainly concentrate on developing software quality.

Before explaining about development of agile first we have to know what is the correct meaning on agile in a single word agile is nothing but adopting continuous changes. Agile can react to changes fast and accurately. Agile involves more on customer participation during development of the product such that customer product is designed accord to the him Thus inventory requires these type of software model to manages it's stocks.

Here inventory is nothing but stocks which is referring to all materials and goods Where inventory management implies control over the stocks/items that are ordered by customers, exchange of stocks, Intermediate components that are used by company in producing the final product, producing to goods to customer time to time. So to convince all these traditional software faces many problems this is the reason why companies chooses agile on inventory management because agile has the flexibility to change requirements and main advantage is testing is done regular bases such that we can save time and we can find faults immediately

Agile in inventory management also helps in minimizing the cost of change in such a way that agile has been defined. As we know that agile has different methodologies in choosing the methodology we compare with each and every one and choose best of them such that in this agile we have chosen agile unified process which is the extension of rational unfired process It mainly focus on flexibility though AUP concentrate on size of process and speed of delivery. The application of inventory management on real time industries can be clearly understand if we know the marketing system of reliance. Reliance is the one which produces electronic goods, slippery, eatables etc. In order to know about different goods it creates different sites 
Inventory Management to Secure Software System

such that it creates a vast marketing in India and other countries also.

\subsection{Additional information}

Inventory can be developed not only by AUP but also with traditional but when comparing the benefits of agile unified process with traditional methods agile unified process has more. agile unified process is more popular for it's incremental and also iterative approach which impels that step by step process.

The next important character is architectural approach because without knowing stock architecture it will be very tough and risk to manage stacks so architectural approach plays a crucial role in managing the stocks and single architecture is not able to solve the all model etching a system. It needed multiple architecture. To handle this multiple architecture only agile unified process is best one. It can support all the architecture models. Agile unified process which is the extension of rational unified process has four phases but difference arrives during solving in agile unified process are construction, incremental etc follows step by step process which ha s mainly 5 steps they are analysis,requirements,design,testing and coding.

It is called as engineer approach because it is the firth approach in software. During traditional approach the main disadvantage is it fails in solving the complex problem.

\section{Software System}

\subsection{Experimental Investigations}

Presently a-days, there is a broad manage giving the security to software frameworks, this is on account of numerous organizations depend for the most part on them for their everyday capacities. As we haven't seen a product framework that is thoroughly secure and can be sensible till now, it is required and important to analyze and find the dangers of developing software frameworks. Alongside the particulars system period generally gained, the security, the information present in the data framework is prepared to confront a test and it is additionally is by all accounts important to assess its danger.

Expressing the nimble, this content investigations the data about the components like time management, software, information management, complexities and costs that is included from framework hazard, system hazard, information administration framework, environment, and operation. This gives the assessed technique for stock framework and infers the danger it 
confronted. The light-footed complete study clarifies stock in point of interest. The model and the technique we picked turned out to be extremely helpful and compelling by genuine case.

The work that is done speaks to a procedure for dissecting programming security utilizing lithe philosophy framework. The inputs that are given to the framework are suitable for speaking to the necessities and outline design for accomplishing great item. While planning it is required to outline in a manner that the last programming give classification, honesty and accessibility which goes about as programming security objectives. The configuration execution is done utilizing net beans and to give security we can utilize Ajax programming. This is on account of the product has capacity to execute online frameworks.

\subsection{Problem or Issue Being Solved}

Today, security issue which is included in PC based frameworks are getting significantly more as often as possible and this calls for genuine mindfulness. The number and different diverse assaults by individual and malevolent programming which are from outside association, especially from the Internet are expanding quickly. The amount and outcomes of internal assaults additionally remain a noteworthy worry in overseeing programming.

In any case, it is acknowledged that the outlining of the secured programming is a troublesome issue generally. Assailants for the most part break into frameworks and accordingly, some stock general store programming merchants had as of now begun giving the security as a fundamental variable for their items and system frameworks. Thus for the results of years of exploration in security of PCs and in overseeing of information most effective systems have been created to tackle a wide exhibit of security and information issues. Aside from this, delivering a product which can likewise be secured furthermore give more classification to the handled information programming items would require a considerable measure of work with respect to the stock procedure. This procedure of execution, has a critical stage which has been risen into the advancement of programming with a specific end goal to enhance the spry bound together process is the stage for testing, which is essentially utilize framework and client testing strategies. The dangers ought to be recognized, positioned, alleviated and afterward it ought to be overseen absolutely through the product item lifecycle. Once the dangers are recognized, modification ought to be made to the product before the last item is sent. Hence, this will propose a coordinated based method for the determination of the level of danger which is connected with programming frameworks. 


\subsection{Data and Evidence}

In spite of the endeavours which are done towards delivering the high calibre and secured programming frameworks, the aggressors still over and over break into a few frameworks. In extra to the reaction gave, programming merchants began consolidating the procurement of security as an essential components for their items. Programming security on information procurement is a basic element to data affirmation furthermore outlining level vulnerabilities are even in charge of up to half of the security issues in coding. Past examination works that are done around there have displayed or proposed diverse sorts of security on information measures and this leads forms towards delivering secured programming. Recognizing the benefits and getting to focuses, and distinguishing the dangers. The dangers related which recognize dangers were then surveyed.

In request to get a product which discharge quicker with high caliber and diminish hazard we can utilize lithe procedure. As of late in uk where there is a most prominent news paper site which indicates 700 pages for each second and the development every year would be around $30 \%$. it began with initiation stage on ventures later expanded its development gradually.

\subsection{Inferences of Data}

The methodology of coordinated has been as of late utilized for the determination of dangers in various circumstances. The strategy of lithe depended on various approaches and the surmising motor which is trying strategies and outline programming was utilized to recognize the dangers to PC based frameworks which are potential. The outcome demonstrates a compelling method for doing the model spoke to as far as chart. A nimble methodology strategy which is intellectual was intended for improving danger in a grocery store frameworks. The work that has been introduced is a strategy for examining and evaluating the dangers brought about in data frameworks of business. In the past papers, the appraisal of danger was completed by considering the marvel of what might happen if a predetermined choice was taken or in the event that some sort of data was lost or it is rendered unauthentic. A guide of customary methodology is utilized to get a photo of the distinctive stages obviously. The issue with in this sort of method is, that conventional methodology is not effectively calculable.

\subsection{Statement of purpose}


The main purpose is as the population in world is getting increased day by day in order to handle the stocks it is becoming impossible to continue the process further because in olden days all the stock record system is usually handled by human manually even now either in cities or towns or villages there are small whole sale or super market shops where we see many people work manually but coming to large super market shops like reliance, more examples, it is becoming impossible to make record of all the goods, to handle the huge people coming to market and purchasing goods, keeping the records of all purchased goods, also keeping the records of finished goods, making sure that delivery of goods is done effectively or not. All these problems can be seen in many small and large scale businesses where there are facing problems to manage and utilize data for the growth rate of their private business.

\subsection{Experimental Results}

The inputs that are given to the framework are the qualities that are gained through necessities and those lead to ascertained for privacy, uprightness and accessibility which are the product security objectives. The objectives are induced to be of the same weight and a specific worth is persevering for each of them predicated on inquiries that are replied about the solid programming. Withal the qualities decided for each of the info are characterized as an in origin stages, later through those qualities outline will be drawn and working will be talked about. Amid this procedure testing happens at various stages and we have numerous testing methods utilizing ny one of the systems we can distinguish dangers. All the above steps can be examined utilizing any nimble approach. Spry is a quicker, solid, and effective model.

Short-term and long-term implications: Agile procedure is mind boggling, multicriteria and typically multilevel choice frameworks, and they are required to deal with the instabilities. Additionally lithe environment can speak to the befuddled danger calculates and leads a worthy structure, where the danger variables can be gathered in view of the diverse developement framework. The framework parameters' association is not reliant on each other in the event that one stage gets destructed amid improvement it doesn't indicate more exertion on different stages that is the reason the nimble methodology are connected with stock frameworks. On the off chance that ones the essential necessities are gathered there can be utilized till the item is done i.e., till definite item. Later in future additionally these prerequisites can be utilized if as a part of case any debate get happened. 


\subsection{Long Term Goals}

It may likewise be important to utilize a versatile dexterous strategy for security and dealing with the information. We have possessed the capacity to outline a framework that can be utilized to assess the danger connected with the creation of secure programming frameworks. This will help programming associations get together with the standard prerequisites. A strategy for surveying security of programming framework before conclusive arrangement has been introduced. The aftereffect of this study demonstrates that if the product creating organizations will consolidate hazard investigation into the generation of programming framework, the issue of frailty of programming will be held to the base if not wiped out. The ought to likewise make a note of the amount of information the product is competent to handle. This study has likewise uncovered that if each of the product security objectives can be expanded to the most extreme, then the level security will likewise be expanded and the danger related will be killed.

At long last, dexterous strategy is a way towards creating secure programming and ought to be viewed as a noteworthy movement by programming improvement associations.

Assumptions behind the inferences, selection or collection of data, or framing of the problem / experiment: To actualize the product for stock administration framework the Agile Unified Process is utilized. It depends on the proof of the Agile strategy which is utilized as a part of the product associations for the improvement. The stock administration application is an iterative and incremental formative application where the prerequisite are recovered in the every emphasis and it ought to be earth overseen by sending it into the web in every stage furthermore information ought to be accounted for at each stage.

\subsection{Software system}

To complete the item for stock organization system the Agile Unified Process is used. It relies on upon the confirmation of the Agile technique which is used as a part of the item relationship for the change. The stock organization application is an iterative and incremental developmental application where the essential are recouped in the each accentuation and it should be earth administered by sending it into the web in each stage moreover data should be represented at every stage. 


\subsection{Software security}

software security is an origination which is executed to rampart the product against the dangerous assault and other programmer hazards so that the product sustains to work accurately under such potential risk. Security is obligatory to give respectability, secrecy and accessibility.

\subsection{Testing levels}

As a rule we have numerous testing levels like unit testing, framework testing, reconciliation testing, alpha testing, beta testing e.t.c. In unit framework usefulness of code at area level is tried. In framework testing we give wrong inputs and test whether framework is working accurately or not. In client testing we request that client test. Correspondingly all other testing.

\subsection{Discussion of Results}

The application can be induced using the Web and database advances like HTML, CSS, JS, PHP, Mysql. The Mysql and PHP are used as the back-end innovations for building up the any sort of use. The interface is produced by utilizing the HTML ,CSS and JScript. The application is can be conveyed in any free facilitating web convenience suppliers. The well known kenned organization dependence built up its product using java.

Like Java, PHP is likewise a server-side scripting dialect which is utilized for outlining web advancement however it is additionally used as a general-imply dialect. Really PHP remains for Personal Home Page, at present it remains as Hypertext Preprocessor. Time administration is essential in any advancement yet in conventional a considerable measure of time get squandered in every level except it doesn't happen in coordinated. Fulfilling the client needs is troublesome in nimble and simple in customary.

\section{Conclusion}

We are portraying our investigation on nimble brought together process and their controls where we clarify how these orders help being developed of stock administration and we will likewise clarify the usefulness of stock, assignments performed in every phase of stock. There is plausibility of this framework to be planned once more. The update ought to be in a manner that it can be utilized on any system not just a specific philosophy called deft procedures. The 
most essential thing is that it ought to be composed in a manner that is deployable effortlessly. This is for stock administration. There is a current configuration framework in which it can assess the danger of security in information that was there in the advancement of programming frameworks which are secure. This will be exceptionally helpful for the associations to keep up their gauges. Security of programming should be checked before the last organization. This gives a strategy through security can surveyed effortlessly.

\section{References}

[1] V. R. Basili, L. Briand, and W. Melo, A Validation of object oriented design metrics as quality indicators in IEEE Transactions on Software Engineering, (1996), Vol.22, No.10, pp.751-761.

[2] http://satc.gsfc.nasa.gov/support/STC_APR98/apply_oo.html, July 25 (2016).

[3] IEEE Standard 1633TM, IEEE Recommended Practice on Software Reliability- IEEE Reliability Society, sponsored by the Standards Committee (2008).

[4] H. L. Wu, Y. Zhong, and Y. Chen, A Software Reliability Prediction Model Based on Benchmark measurement, IEEE International Conference on Information management and Engineering, (2011) April, pp.131-134.

[5] R. Roshandel, S. Banerjee, L. Cheung, N. Medvidivic, and L. Golubchik, Estimating Software Component Reliability by Leveraging Architectural Models, ACM ICSE'06, (2006) May 20-28; Shanghai, China.

[6] E. E. Ozguven and K. Ozbay, A secure and efficient inventory management system for disasters, Transportation Research Part C, (2013), Vol.29, pp.171-196.

[7] B. M. Beamon and S. A. Kotleba, Inventory modelling for complex emergencies in humanitarian relief operations, International Journal of Logistics: Research and Applications, (2006), Vol.9, No.1, pp.1-18.

[8] M. Brodie, E. Weltzien, D. Altman, R. J. Blendon, J. M. Benson, Experiences of hurricane Katrina evacuees in Houston shelters: implications for future planning. American Journal of Public Health, (2006), Vol.96, No.5, pp.1402-1408.

[9] H. X. Liu, X. J. Ban, W. Ma, and P. B. Mirchandani, Model reference adaptive control framework for real-time traffic management under emergency evacuation, Journal of Urban Planning and Development, (2007), Vol.133, No.1, pp.43-50. 